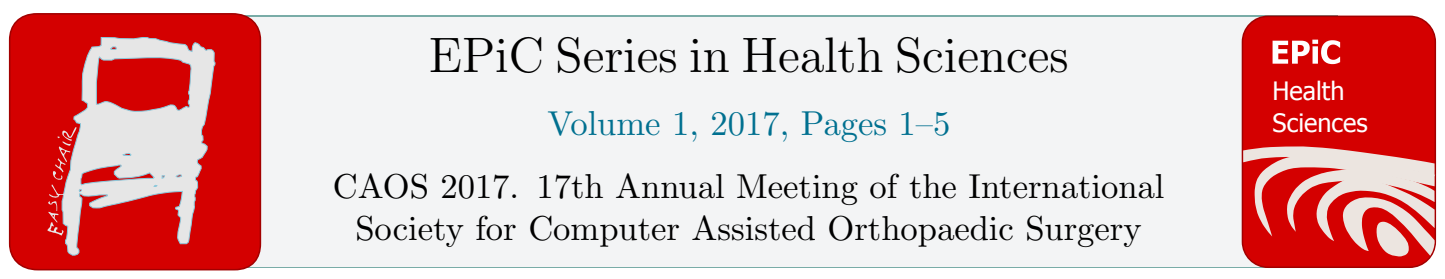

\title{
The Direct Repair of Unilateral Spondylolysis with the Guidance Aid of a Personalised Rapid Prototyping Template
}

\author{
Janez Mohar \\ Valdoltra Orthopaedic Hospital, Slovenia \\ janez.mohar@ob-valdoltra.si
}

\begin{abstract}
A case of an 18-year-old professional soccer goalie, diagnosed with a unilateral isthmic defect of L5 vertebra is presented. The isthmic stress fracture was stabilised by a lag screw fixation (i. e. Buck's technique) with the drill bit guidance of a personalised rapid prototyping 3D template. A high degree of screw placement accuracy with this simplified modification of the technique is reported.
\end{abstract}

\section{Introduction}

The reported incidence of spondylolysis in athletes varies between $15 \%$ and $47 \%$, considerably more than $6 \%$ in the general population, with most common types of related sport activities being gymnastics, weight lifting, soccer, swimming, cricket and tennis. (Reitman, Stanitski). The repeated tension stress microtrauma on the ventro-caudal part of the inferior articular facet during lumbar hyperextension and trunk twisting movements, together with anatomical predispositions can result in complete symptomatic fracture defect, preventing further participation in sports activities. Although less common and more benign, unilateral isthmic stress fractures tend to be resistant to conservative management if not properly addressed initially, therefore warranting early operative treatment (Debnath).

\section{Materials and Methods}

The aim of this paper is to report a case of a lag screw repair of a unilateral spondylolysis with a guidance aid of a patient specific rapid prototyping 3D template.

An otherwise healthy 18 -year-old professional soccer goalie experienced a spontaneous, right lower back pain after intensive practice that was non progressive, but severe enough to prevent him from involvement in strenuous physical activity. The MRI revealed a bony edema of the right L5 pedicle 
and a CT scan was performed in order to distinguish a post traumatic lesion from a possible occurrence of an osteoid osteoma. A definitive diagnosis of an unilateral right isthmic defect was therefore confirmed and an operative treatment of a direct lag screw fixation (modified Buck's technique) was offered to him, since this type of osteosynthesis is considered to be biomechanically superior to other spondylolysis repair techniques like Scott wiring or Morscher's hook-screw system. He instead opted for a 3-month immobilisation in a thoracolumbar right-sided unilateral above-knee hip spica cast after which a controlled CT scan revealed no signs of bone healing, in fact fracture lines were smoother and more sclerotic. The preoperative image analysis offered no margin of error in placement of a $3.5 / 35 \mathrm{~mm}$ lag screw in all three planes so a decision to use a trajectory guidance aid of a personalised rapid prototyping $3 \mathrm{D}$ template was made (Figure 1).
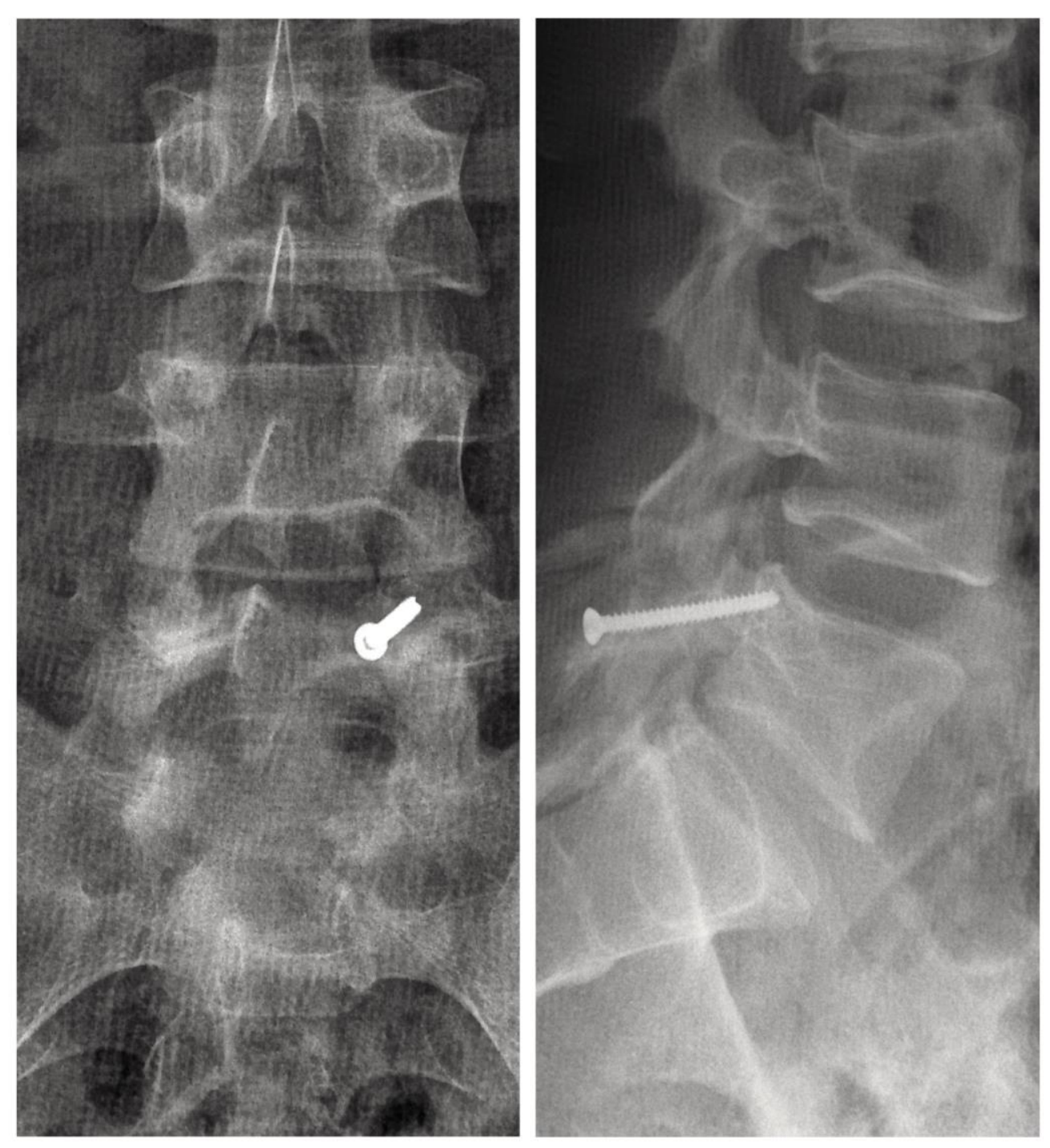

Figure 1: Postoperative X-ray of the patient's lumbar spine showing a lag screw transversing the right pars interarticularis 


\section{Results}

The patient's clinical postoperative course was uneventful, follow-up imaging showed signs of bone healing (Figure 2). One year after the operation he was able to return to his previous level of sports activity and he currently plays for a first national league soccer club as a reserve goalie.
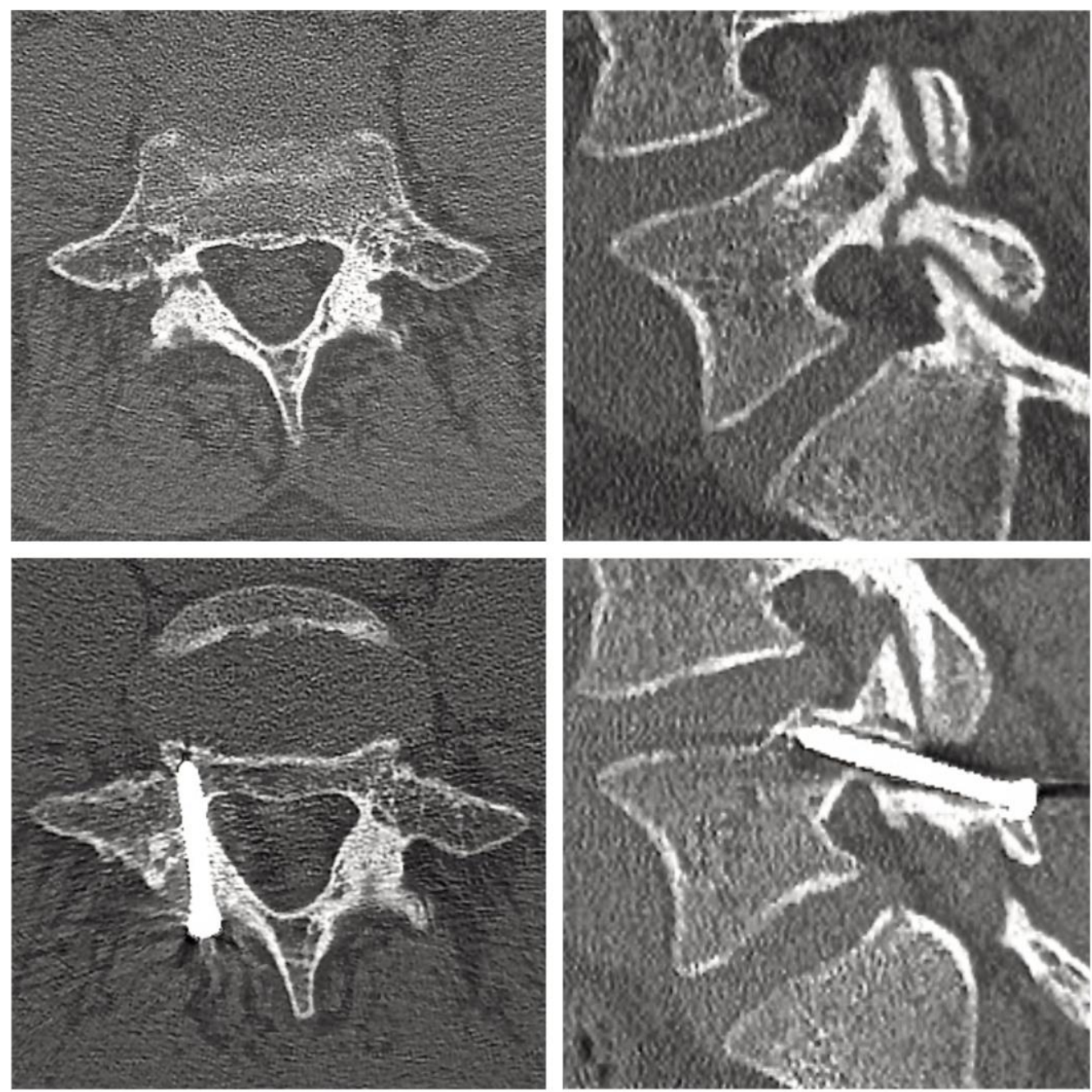

Figure 2: Pre- and postoperative CT images of the L5 vertebra. Note the perfect position of the lag screw, perpendicular to the fracture line and the proximity of the spinal canal, intervertebral foramen and disc space. 
The comparison of planned and actual lag screw position showed that the entry point offset on oblique coronal plane was $0.2 \mathrm{~mm}$ and $1.3 \mathrm{~mm}$ on $\mathrm{X}$ and $\mathrm{Y}$ axis, respectively. The difference in trajectory angle was $0.2^{\circ}$ and $0.5^{\circ}$ on oblique sagittal and oblique transverse plane, respectively (Figure 3).
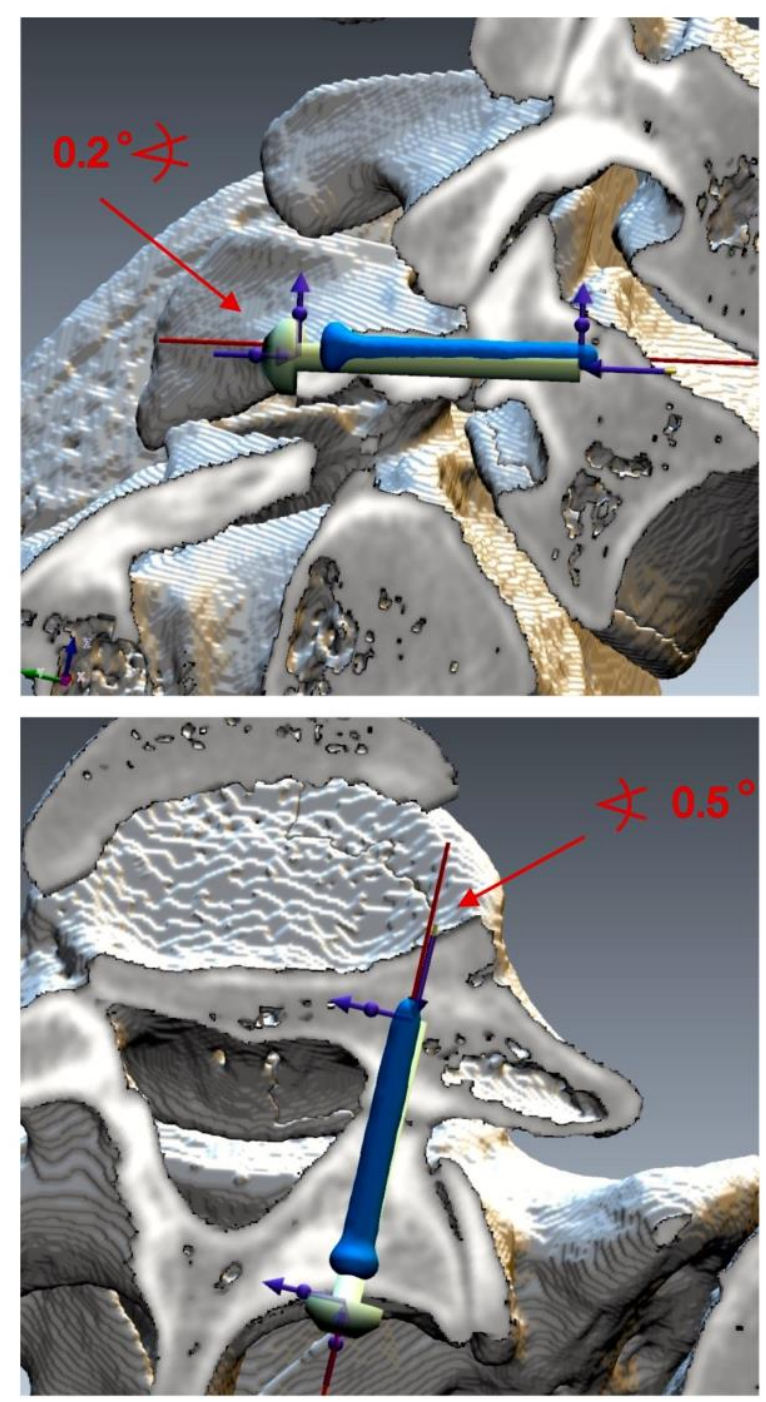

Figure 3: Preoperative planning position of a lag screw (green) and postoperative position (blue) on oblique sagittal and oblique transverse plane

\section{Discussion}

A personalised 3D template, used as a guidance aid in Buck's repair, allows a higher degree of screw placement accuracy and simplifies the procedure compared to conventional technique 
(Rajasekaran, Snyder). To the best of author's knowledge, this is the first reported case of a direct spondylolysis repair with the use of patient specific rapid prototyping technology.

\section{References}

Reitman CA, Esses SI, Direct repair of spondylolytic defects in young competitive athletes, Spine J. 2002;2(2):142-4.

Stanitski CL, Spondylolysis and spondylolisthesis in athletes, Oper Tech Sports Med. 2006;14(3):141-6.

Debnath UK, Freeman BJC, Grevitt MP, Sithole J, Scammell BE, Webb JK, Clinical outcome of symptomatic unilateral stress injuries of the lumbar pars interarticularis, Spine 2007;32(9):995-1000.

Rajasekaran S, Subbiah M, Shetty AP, Direct repair of lumbar spondylolysis by Buck's technique, Indian J Orthop. 2011;45(2):134-40.

Snyder LA, Shufflebarger H, O'Brien MF, Thind H, Theodore N, Kakarla UK, Spondylolysis outcomes in adolescents after direct screw repair of the pars interarticularis, J Neurosurg Spine 2014:21(3):329-33.

\section{Disclosures}

The manuscript submitted does not contain information about medical device(s)/drug(s). No funds were received in support of this work. The author reports no relevant financial activities outside the submitted work and declares no conflict of interest. 